\title{
Evaluating Korean Personal Assistance Services Classification System
}

\author{
Mi Jung Lee, PhD $^{1,2}$, Sergio Romero, PhD $^{1,2}$, Ickpyo Hong, $\mathrm{PhD}^{3}$, Hae Yean Park, $\mathrm{PhD}^{4}$
}

\begin{abstract}
${ }^{1}$ Department of Occupational Therapy, University of Florida, Gainesville, FL, USA; ${ }^{2}$ Center of Innovation on Disability \& Rehabilitation Research (CINDRR), North Florida/South Georgia Veterans Health System, Gainesville, FL, USA; ${ }^{3}$ Department of Occupational Therapy, University of Texas Medical Branch, Galveston, TX, USA; ${ }^{4}$ Department of Occupational Therapy, Yonsei University, Wonju, Korea
\end{abstract}

\begin{abstract}
Objective To evaluate the utility of using the Personal Assistance Services classification system (PAS-CS) that examines individuals with disabilities for services and government funding. To this end, this study also tests for significant differences in PAS-CS scores across disability grades and disability types.

Methods A retrospective analysis was conducted using the 2014 National Survey on People with Disabilities (NSPD) data set. We selected patients with three types of disabilities (physical disabilities, brain lesions, and visual impairments). We compared the average PAS-CS scores of patients with different disability types and grades using general linear models with multiple comparisons.

Results A total of 4,810 patients were included in the analysis. Patients with brain lesions had the highest average PAS-CS scores in activities of daily living (ADL) and instrumental activities of daily living (IADL) domains. Patients with visual impairments had the highest average scores in 'Disease-specific disability' and 'Social-environment' domains. For patients with physical disabilities and visual impairments, no PAS-CS domains were significantly different between patients with disability grade III and those with disability grade IV ( $p>0.05)$.

Conclusion The PAS-CS scores of disability grades were not equivalent among individuals with different disability types. The Korean Ministry of Health and Welfare currently only considers certain disability grades for PAS preeligibility, as a result disregarding the characteristics of different disability types. Thus, the current PAS-CS requires modifications.
\end{abstract}

Keywords Disability evaluation, Public assistance, Social welfare, Republic of Korea

\section{INTRODUCTION}

As part of each person's non-negotiable civil and politi- cal rights, Personal Assistance Services (PAS) are social services that support individuals with disabilities through providing assistance to help them cope with daily activi-

Received November 15, 2017; Accepted January 25, 2018

Corresponding author: Mi Jung Lee

Department of Occupational Therapy, University of Florida, 1225 Center Drive, Suite 2101, Gainesville, FL 32611, USA. Tel: +1-352-273-6817, Fax: +1352-273-6042,E-mail: mjlee1@ufl.edu

ORCID: Mi Jung Lee (http://orcid.org/0000-0002-2753-8105); Sergio Romero (http://orcid.org/0000-0001-7379-7434); Ickpyo Hong (http://orcid. org/0000-0001-5404-7646); Hae Yean Park (http://orcid.org/0000-0003-0822-9902).

두 This is an open-access article distributed under the terms of the Creative Commons Attribution Non-Commercial License (http://creativecommons.org/ licenses/by-nc/4.0) which permits unrestricted noncommercial use, distribution, and reproduction in any medium, provided the original work is properly cited. Copyright (c) 2018 by Korean Academy of Rehabilitation Medicine 
ties and demands, and ultimately enhancing their participation in society [1-4]. These services are designed to meet each individual's specific needs, so that he/she may complete the specific day-to-day tasks that are necessary for them $[2,5]$.

PAS can vary in the forms they take, such as providing guidance, hands-on assistance, and emotional support $[2,5]$. As PAS can potentially enable individuals with disabilities to live more independent lives, maintain their careers, and participate in their communities, a lack of support or inappropriate support prescription can decrease patients' quality of life, worsen their health and disabilities, lead to institutionalization, and even cause death $[1,5-8]$.

Since 2011, the Korean Ministry of Health and Welfare has provided nationwide PAS to individuals with severe disabilities in order to help them maintain independence $[4,9]$. Each individual's need for PAS varies depending on their levels of disability and social function, which means that each individual requires different allocations of financial support in order to pay for his/her respective services. Primarily, the Personal Assistance Services classification system (PAS-CS) is used to determine what PAS level an individual requires.

The purpose of PAS is to improve social participation of patients with severe conditions [9]. The current PASCS was created based on two pilot projects, conducted in 2008 and 2010 [4]. On the first pilot test, multiple instruments used by locally funded PAS and long-term care services were tested [4]. The discrepancies among instruments raised issues in interpreting and comparing patients' PAS needs [4]. Thus, the second pilot project developed and tested a single integrated PAS-CS form. However, this form was criticized for being analogous to the long-term care assessment instrument, which focuses on supporting elderly individual's daily activities and alleviating caregiver burdens in their households $[4,10]$. In 2011, PAS-CS was revised by focusing on activities of daily living (ADL) and instrumental activities of daily living (IADL) as central concepts, since ADL and IADL have been effectively used to evaluate PAS needs to enhance patients' social participation in the United States, Japan, Germany, and Sweden [4].

The PAS-CS includes four major domains: ADL (total score of 260), IADL (total score of 125), Disease-specific disability (total score of 60 ), and Social-environment (total score of 25). Summed scores (up to 470) are used to determine PAS levels, with higher scores indicating greater needs for PAS. Registered evaluators visit the applicant's homes and assess his/her conditions using the PAS-CS form and personal interviews.

Based on the given scores, the Korean Ministry of Health and Welfare determines the level of PAS for that particular individual. PAS-CS categorizes individuals into four levels: level 1 (380-470), level 2 (320-379), level 3 (260-319), and level 4 (220-259). For individuals with PAS-CS scores below 220, no PAS are provided, with the exception of patients with special needs, such as those who are pregnant, without caregivers, or attending school [9]. The amount of monthly financial support for the four PAS levels are as follows: KRW 1,091,000 for level 1; KRW 869,000 for level 2; KRW 657,000 won for level 3; and KRW 435,000 for level 4. Additional support can be provided for individuals with special needs such as those listed above.

It is still questionable whether the current PAS-CS is the most useful and beneficial tool for assessing the needs of people with disabilities. The eligibility criteria include: (1) being registered in the government system as an individual with a disability, (2) age from 6 to 65 years old, and (3) holding a disability grade of I, II, or III. This means that regardless of their needs, individuals with disability grades IV through VI cannot even apply for PAS. Disability grades are primarily determined by a patient's medical condition(s), which may not fully reflect his/her needs for PAS. It has yet to be confirmed that individuals with disability grade III have statistically greater PAS-CS scores than those with disability grade IV. While this topic requires further investigation, if no statistical differences exist between disability grades III and IV, PAS-CS's prerequisite criteria for PAS must be modified. Unlike in Korea, Japan allows individuals of all disability grades to apply for PAS, and applicants are assessed using 106 items before being provided the services deemed necessary [4].

In 2015 , three disability types-physical disabilities, brain lesions, and visual impairments-accounted for $71.8 \%$ of $2,490,406$ individuals registered with a disability in Korea [11]. These three disability types encompass a wide range of physical functions and account for the majority of individuals with disabilities; thus, they are ideal types for investigating PAS needs for different disability types and grades. 
The purpose of this paper is to (1) compare each PASCS domain (ADL, IADL, Disease-specific disability, and Social-environment) of different disability types and grades, and (2) to investigate whether or not there is a significant difference in PAS-CS scores between patients with disability grades III and IV.

\section{MATERIALS AND METHODS}

\section{Data collection}

Data was retrieved from the 2014 National Survey on People with Disabilities (NSPD), which examines the disability status of the Korean population (Korean Institute for Health and Social Affairs, 2014 [12]. Trained surveyors visited 200 survey participants per approximately 45,000 households in order to collect this data set. The data includes information on: basic demographics, economic status, health, and use of health care resources. This analysis used PAS-CS responses for patients with three disability types (physical disabilities, brain lesions, and/ or visual impairments). This study was exempted by the Institutional Review Board of University of Texas Medical Branch at Galveston (No. 18-0241).

\section{Personal Assistance Services classification system}

Different numbers of items are included in four domains of PAS-CS: ADL has 7 items, IADL has 8 items, Disease-specific disability has 5 items, and Social-environment has 4 items. The scores given to each item are different: for example, total dependency in dressing has a score of 40 , whereas total dependency in bathing has a score of 30 . We totaled the PAS scores of all four domains following the current PAS-CS scoring system. Detailed information on the items and their scores is provided in Table 1.

\section{Statistical analysis}

This paper used two statistical procedures: descriptive statistics and inferential statistics. For descriptive statistics, we calculated average scores for the disability types and grades, which were summarized into overall scores as well as the scores for each PAS-CS domain. Then, using multiple comparisons via general linear models, we tested statistical inferences in PAS-CS scores among different disability grades for different disability types (disability grade III vs. disability grades IV, see Equation 1).
We also tested assumptions for general linear models, such as normality and homoscedasticity of errors.

$$
\begin{aligned}
& \text { PAS scores }{ }_{i}=\beta_{0}+\beta_{1} \text { brain }_{i}+\beta_{2} \text { visual }_{i}+\beta_{3} \text { gII }_{i}+\beta_{4} g I I I_{i}+\beta_{5} g I V_{i} \\
& +\beta_{6} g V_{i}+\beta_{7} g I_{i}+\beta_{8} \text { brain }_{i}^{*} \text { gII }_{i}+\beta_{9} \text { visual }_{i}^{*}{ }^{*} \text { III }_{i}+\beta_{10} \text { brain }_{i}^{*} \text { gIII }_{(1)} \\
& +\beta_{11} \text { visual }_{i}^{*}{ }^{*} \text { III }_{i}+\beta_{12} \text { brain }_{i}^{*}{ }^{*} \text { gIV }_{i}+\beta_{13} \text { visual }_{i}^{*}{ }^{*} \text { gIV }_{i}+\beta_{14} \text { brain }_{i}^{*}{ }^{*} \\
& \mathrm{gV}_{i}+\beta_{15} \text { visual }_{i}^{*} \mathrm{gV}_{i}+\beta_{16} \text { brain }_{i}^{*}{ }^{*} \mathrm{gVI}_{i}+\beta_{17} \text { Visual }_{i}{ }^{*} \mathrm{gVI}{ }_{i}
\end{aligned}
$$

where $\beta=$ coefficients, brain=brain lesions, visual=visual impairments, g=disability grades.

For inferential statistics, we randomly selected 300 individuals for each grade ( $\mathrm{n}=1,800$ for 6 disability grades) in order to prevent disproportionate results between the disability grades. Statistical programs R, RStudio version 1.0.136 (R Core Team, Cary, Vienna, Austria), and various packages (lavaan, dplyr, MuMIn multicomp, AER, moments, gvlma, and MASS) were used for all computational processes [13-21].

\section{RESULTS}

\section{Demographic information}

Among 6,401 patients selected for analyses, 4,810 (75.1\%) patients had one of the three selected disability types: 3,475 (72.2\%) had physical disabilities, 687 (14.3\%) had brain lesions, and 648 (13.5\%) had visual impairments. Of the 4,810 patients, 2,659 (55.3\%) were male and $2,151(44.7 \%)$ were female. The mean age in this sample was $62.6 \pm 14.2$ years. More than half of patients (54.4\%) were patients with disability grades V and VI. More information regarding our sample sizes for each disability group and grade is provided in Table 2 .

\section{Descriptive statistics}

The average scores for all three disability types and 6 disability grades are provided in Table 3 .

\section{Overall PAS-CS scores}

Out of a total PAS-CS score of 470, on average, patients with brain lesions grade I had the highest overall PAS-CS scores (320.9), followed by patients with visual impairments grade I (232.5) and physical disabilities grade I (220.5) in order. The average scores indicate that patients with brain lesions grade I are eligible for PAS-CS level 2 while patients with physical disabilities and visual impairments grade I are eligible for PAS-CS level 4. Beyond 
Table 1. PAS-CS items

\begin{tabular}{|c|c|c|c|c|c|c|}
\hline \multirow{2}{*}{$\begin{array}{ll} & \text { Domain } \\
\mathrm{ADL}\end{array}$} & \multirow[b]{2}{*}{ Dressing } & \multicolumn{4}{|c|}{$\begin{array}{c}\text { Levels of dependency } \\
\text { (Scores) }\end{array}$} & \multirow{2}{*}{\begin{tabular}{l}
\multicolumn{1}{c}{ Comment } \\
Max score $=260$ \\
Sum scores of all 7 items
\end{tabular}} \\
\hline & & 0 & 10 & 20 & 40 & \\
\hline & Bathing & 0 & 10 & 20 & 30 & \\
\hline & Eating & 0 & 30 & 60 & 90 & \\
\hline & Positioning in bed & 0 & 10 & 15 & 20 & \\
\hline & Transferring & 0 & 10 & 15 & 20 & \\
\hline & Walking & 0 & 10 & 20 & 30 & \\
\hline & Toileting & 0 & 10 & 20 & 30 & \\
\hline \multirow[t]{8}{*}{ IADL } & Using telephone & 0 & 5 & 10 & 15 & $\begin{array}{l}\text { Max score }=125 \\
\text { Sum scores of all } 8 \text { items }\end{array}$ \\
\hline & Purchasing* & 0 & \multicolumn{2}{|c|}{10} & 15 & \\
\hline & Preparing a meal & 0 & 10 & 20 & 30 & \\
\hline & Managing household chores & 0 & 5 & 10 & 15 & \\
\hline & Doing laundry & 0 & 5 & 10 & 15 & \\
\hline & Taking medication* & 0 & \multicolumn{2}{|c|}{10} & 15 & \\
\hline & Managing financials* & 0 & \multicolumn{2}{|c|}{5} & 10 & \\
\hline & Taking public transportation & 0 & 3 & 5 & 10 & \\
\hline \multirow[t]{5}{*}{ Disease $^{b)}$} & Using wheelchair* & 0 & \multicolumn{2}{|c|}{15} & 30 & $\begin{array}{l}\text { Max score }=60 \\
\text { Apply only one item's score }\end{array}$ \\
\hline & Auditory ability & 0 & 20 & 40 & 60 & \\
\hline & Visual ability & 0 & 20 & 40 & 60 & \\
\hline & Cognitions* & 0 & & 0 & 60 & \\
\hline & Mental conditions* & 0 & & 0 & 60 & \\
\hline \multirow[t]{4}{*}{ Social-environment } & Social participation* & 0 & & 5 & 10 & $\begin{array}{l}\text { Max score }=25 \\
\text { Sum scores of all } 4 \text { items }\end{array}$ \\
\hline & Management of emergent situations* & 0 & & 3 & 5 & \\
\hline & Using assistive device independently* & 0 & & 3 & 5 & \\
\hline & Independent communication* & 0 & & 3 & 5 & \\
\hline
\end{tabular}

PAS-CS, Personal Assistance Services classification system; ADL, activities of daily living; IADL, instrumental activities of daily living.

Items with * have only three rating levels.

${ }^{a)}$ Higher scores indicate higher dependency in performing items.

${ }^{b}$ Disease indicates 'Disease-specific disability' domain.

Table 2. Number of participants for each disability type and grade

\begin{tabular}{lrrrrrrr}
\hline \multirow{2}{*}{ Disability type } & \multicolumn{4}{c}{ Disability grade } & \multicolumn{1}{c}{ V } & VI & Total \\
\cline { 2 - 7 } & I & II & III & IV & 721 & 1035 & 993 \\
\hline Physical disabilities & 89 & 193 & 444 & 9475 \\
Brain lesions & 124 & 137 & 190 & 92 & 76 & 68 & 687 \\
Visual impairments & 106 & 29 & 38 & 28 & 60 & 387 & 648 \\
\hline
\end{tabular}

these groups with grade I disabilities, no other groups were eligible for any levels of PAS-CS on average.

\section{ADL PAS-CS scores}

Out of a total score of 260, patients with brain lesions 
Table 3. Average PAS-CS scores for disability types and grades

\begin{tabular}{|c|c|c|c|c|c|c|c|}
\hline \multirow{2}{*}{ Domain } & \multirow{2}{*}{ Disability type } & \multicolumn{6}{|c|}{ Disability grade } \\
\hline & & I & II & III & IV & V & VI \\
\hline \multirow[t]{3}{*}{ Overall } & Physical disabilities & 220.51 & 98.70 & 49.48 & 33.59 & 24.86 & 15.40 \\
\hline & Brain lesions & 320.85 & 215.66 & 141.55 & 107.28 & 65.00 & 65.37 \\
\hline & Visual impairments & 232.45 & 129.31 & 119.21 & 81.61 & 61.75 & 43.82 \\
\hline \multirow[t]{3}{*}{$\mathrm{ADL}$} & Physical disabilities & 110.06 & 43.39 & 19.45 & 11.17 & 7.39 & 2.66 \\
\hline & Brain lesions & 174.07 & 105.77 & 63.13 & 46.96 & 23.09 & 26.62 \\
\hline & Visual impairments & 79.95 & 38.97 & 39.21 & 20.18 & 8.00 & 5.01 \\
\hline \multirow[t]{3}{*}{ IADL } & Physical disabilities & 72.87 & 34.74 & 16.84 & 11.60 & 8.25 & 4.24 \\
\hline & Brain lesions & 101.41 & 77.19 & 53.79 & 41.41 & 27.30 & 24.93 \\
\hline & Visual impairments & 81.04 & 37.93 & 34.87 & 22.50 & 16.25 & 8.22 \\
\hline \multirow[t]{3}{*}{ Special $^{\text {a) }}$} & Physical disabilities & 24.94 & 10.49 & 4.77 & 2.74 & 1.30 & 0.56 \\
\hline & Brain lesions & 30.93 & 19.67 & 13.74 & 9.51 & 5.07 & 5.15 \\
\hline & Visual impairments & 54.76 & 38.28 & 33.68 & 28.93 & 27.33 & 21.86 \\
\hline \multirow[t]{3}{*}{ Social-environment } & Physical disabilities & 12.64 & 10.08 & 8.42 & 8.09 & 7.93 & 7.94 \\
\hline & Brain lesions & 14.44 & 13.03 & 10.89 & 9.40 & 9.54 & 8.68 \\
\hline & Visual impairments & 16.70 & 14.14 & 11.45 & 10.00 & 10.17 & 8.73 \\
\hline
\end{tabular}

PAS-CS, Personal Assistance Services classification system; ADL, activities of daily living; IADL, instrumental activities of daily living.

${ }^{\text {a)} S p e c i a l ~ a r e a ~ i n d i c a t e s ~ ' D i s e a s e-s p e c i f i c ~ d i s a b i l i t y ' ~ d o m a i n . ~}$

grade I had the highest ADL PAS-CS scores (174.1), followed by patients with physical disabilities grade I (110.1) and patients with brain lesions grade II (105.8) in order. Notably, patients with brain lesions grade II received higher scores (by 25.8) than patients with visual impairments grade I.

\section{IADL PAS-CS scores}

Out of a total score of 125 , patients with brain lesions grade I had the highest IADL PAS-CS scores (101.4). Patients with visual impairments grade I had the secondhighest (81.0) scores and patients with brain lesions grade II came in third (77.2). It was noted that the average IADL scores for patients with brain lesions grade II were higher (by 4.3) than for those with physical disabilities grade I.

\section{Disease-specific disability PAS-CS scores}

In contrast to the previous results, out of a total score of 60 , patients with visual impairments grade I received the highest Disease-specific disability PAS-CS scores (54.8). Patients with brain lesions grade I (30.9) and physical disabilities grade I (24.9) followed in order.

\section{Social-environment PAS-CS scores}

The scores assigned for this area were relatively smaller than those for the other areas (total score of 25). Patients with visual impairments grade I had the highest scores (16.7). Patients with brain lesions scored the secondhighest (14.4), closely followed by patients with visual impairments grade II (14.1). Patients with brain lesions grade II scored the fourth-highest (13.0), followed in fifth place by patients with physical disabilities grade I (12.6).

\section{Inferential statistics}

We used a randomly selected sample of 1,800 patients (300 patients for each disability grade) for inferential analyses. Assumptions such as normal distribution and homoscedasticity of errors were met. General linear model analyses were used for dependent variables (i.e., overall PAS, IADL, ADL, Disease-specific disability, Social-environment) with the two independent variables (three disability types and six disability grades). The results indicated that $53 \%$ of variance in overall PAS-CS scores was explained by the disability types and grades. Table 4 shows the summary statistics for the model. We focused on examining significant differences between disability grade III and IV for each disability type. De- 
tailed results are shown in Table 5.

For patients with physical disabilities and visual impairments, there was no significant difference between disability grade III and grade IV in overall PAS-CS scores ( $\mathrm{p}=0.08$ and $\mathrm{p}=0.24$, respectively) and ADL PAS-CS do-

Table 4. Model results (overall PAS-CS scores)

\begin{tabular}{|c|c|c|c|c|}
\hline Coefficient & Estimate & SE & $\mathbf{t}$ & p-value \\
\hline$\left.\beta_{0}(\text { Intercept })^{a}\right)$ & 225.18 & 9.61 & 23.43 & $<0.001^{* * *}$ \\
\hline$\beta_{1}(\text { Brain })^{b}$ & 99.57 & 12.65 & 7.87 & $<0.01^{* *}$ \\
\hline$\beta_{2}(\text { Visual) })^{c}$ & 11.79 & 13.10 & 0.90 & 0.37 \\
\hline$\beta_{3}(\mathrm{G} 2)$ & -125.69 & 11.83 & -10.63 & $<0.001^{* * *}$ \\
\hline$\beta_{4}(\mathrm{G} 3)$ & -171.83 & 11.40 & -15.08 & $<0.001^{* * *}$ \\
\hline$\beta_{5}(\mathrm{G} 4)$ & -193.95 & 11.11 & -17.46 & $<0.001^{* * *}$ \\
\hline$\beta_{6}(\mathrm{G} 5)$ & -201.17 & 11.01 & -18.27 & $<0.001^{* * *}$ \\
\hline$\beta_{7}(\mathrm{G} 6)$ & -209.82 & 11.50 & -18.25 & $<0.001^{* * *}$ \\
\hline$\beta_{8}$ (Brain*G2) & 18.24 & 16.72 & 1.09 & 0.28 \\
\hline$\beta_{9}$ (Visual $\left.^{*} \mathrm{G} 2\right)$ & 26.61 & 22.83 & 1.17 & 0.24 \\
\hline$\beta_{10}($ Brain*G3) & -4.05 & 17.39 & -0.23 & 0.82 \\
\hline$\beta_{11}$ (Visual*G3) $^{*}$ & 77.98 & 26.45 & 2.95 & $<0.01^{* *}$ \\
\hline$\beta_{12}($ Brain*G4) & -36.43 & 19.81 & -1.84 & 0.07 \\
\hline$\beta_{13}\left(\right.$ Visual $\left.^{*} \mathrm{G} 4\right)$ & 17.61 & 34.41 & 0.51 & 0.61 \\
\hline$\beta_{14}($ Brain*G5) & -37.80 & 28.15 & -1.34 & 0.18 \\
\hline$\beta_{15}$ (Visual* $^{*}$ G5) & 13.87 & 26.90 & 0.52 & 0.61 \\
\hline$\beta_{16}($ Brain*G6) & -63.25 & 25.22 & -2.51 & $<0.05^{*}$ \\
\hline$\beta_{17}$ (Visual*G6) & 18.32 & 17.43 & 1.05 & 0.29 \\
\hline
\end{tabular}

PAS-CS, Personal Assistance Services classification system; SE, standard error; G, disability grade.

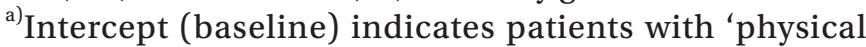
disabilities' with disability grade I, ${ }^{\text {b) }}$ brain lesions, ${ }^{\text {c) }}$ visual impairments.

${ }^{*} \mathrm{p}<0.05,{ }^{* *} \mathrm{p}<0.01,{ }^{* * *} \mathrm{p}<0.001$. main scores ( $\mathrm{p}=0.12$ and $\mathrm{p}=0.20$, respectively). There was no statistically significant difference between disability grade III and IV for all three disability types for the rest of the domains, either (see Table 5).

\section{DISCUSSION}

Disability grades are used as a pre-requisite to apply for PAS. Only individuals with disability grade III and lower (lower grade indicates higher disability) are eligible for PAS. Based on this, it would be expected that significant differences in PAS-CS scores exist between patients with disability grades III and IV. There is an underlying assumption that disability grades can separate individuals into distinct needs categories. However, our study results show that patients with disability grade III are not statistically different from patients with disability grade IV in the majority of PAS-CS domains for all three disability types reported in our study.

PAS needs are not equivalent across disability grades for different disability types, which proves that disability grades should not be considered as a stand-alone criteria. Our study results suggest that patients with different disability types receiving the same disability grade differ in all PAS domains (ADL, IADL, Disease-specific disability, and Social-environment). For example, in patients with brain lesions receiving a grade of III, the ADL, IADL, and Disease-specific disability domain and overall score were anywhere from two to three times higher than in patients with physical disabilities of the same grade. A patient with a disability grade of IV or higher may still have functional limitations that influence his/her ability to function independently. Therefore, in addition to

Table 5. PAS-CS score comparisons

\begin{tabular}{lccc}
\hline & Physical disabilities & Brain lesions & Visual impairments \\
\hline Difference between disability grade III and IV & & & \\
Overall & 0.08 & $<0.05^{*}$ & 0.24 \\
ADL & 0.12 & $<0.05^{*}$ & 0.20 \\
IADL & 0.15 & 0.08 & 0.09 \\
Disability-specific disability & 0.44 & 0.34 & 0.92 \\
Social-environment & 0.89 & 0.46 & 0.83 \\
\hline
\end{tabular}

PAS-CS, Personal Assistance Services classification system; ADL, activities of daily living; IADL, instrumental activities of daily living.

${ }^{*} \mathrm{p}<0.05,{ }^{* *} \mathrm{p}<0.01,{ }^{* * *} \mathrm{p}<0.001$. 
disability grades, disability types should be taken into account when pre-screening patients for PAS-CS application eligibility.

After patients with disabilities meet all of the conditions required to apply for PAS, they may still not receive sufficient PAS-CS scores to receive compensation. In order to receive the minimum level of support of $435,000 \mathrm{KRW} /$ month (PAS level 4), patients are required to obtain a score of 220 PAS-CS or more. This minimum required PAS-CS score might prevent patients with disabilities from receiving needed PAS benefits. Our results show that PAS-CS average scores of patients with disability grade III were $49.5,141.6$, and 119.2 for patients with physical disabilities, brain lesions, and visual impairments, respectively. These scores are far below the required scores for compensation. On average, only patients with disability grade I were considered to be eligible for PAS. This suggests that the required PAS-CS scores might be too high for widespread compensation of PAS. For instance, according to reports released by Seoul Metropolitan Government, among 391,027 registered individuals with disabilities in Seoul, only 15,135 individuals received PAS in 2016 [22,23]. This indicates that required PAS-CS scores may need to be modified if we want to ensure that all individuals with disabilities in need of assistance receive this important benefit.

It is notable that the scores of the Disease-specific disability domain demonstrated different patterns than those of the ADL and IADL domains. The Disease-specific disability domain only considers five impairments: mobility restrictions requiring wheelchair use, diminished auditory, vision, cognitive, and mental function. Thus, other disabling impairments that are not included in this list may be considered disadvantaged [4]. Our results align with this notion: for instance, while individuals in the visual impairment grade III received an average score of 33.7, individuals in the physical disabilities and visual impairments grade III obtained average scores of only 4.8 and 13.7, respectively, for the Disease-specific disability domain. Since this score determines the level of financial support, our data shows that the current system favors specific impairments while not considering other individuals that may be in need of additional support. This requires further investigation at the individual level and may require policy changes.

Our results also indicate potential problems with the
Social-environment domain. While the hierarchy is largely maintained in disability grades across disability types for this domain, the magnitudes of the differences are much smaller than those observed in other domains. For example, the average score difference (across disability type) between disability grades I and VI for the Social-environment domain was 6.14 points compared to a difference in the ADL domain of 109.93. Therefore, the contribution of this domain to the overall score is questionable.

Byun et al. [4] have questioned the ability of the PASCS to fully consider the interactions between individuals with disabilities and the environment. The only items considered in this domain address patient's participation in social events as well as their independent management of emergency situations, use of assistive devices, and communication. Other well-known issues related to person-environment interactions, such as transportation barriers, employment, or education, are not addressed in this domain. Van der Ploeg et al. [24] and Stancil [25] interpreted individual functioning as a person's dynamic interactions or relationships that consistently adapt with personal and environmental factors. For example, a person that is wheelchair dependent may be physically able to drive the chair but may be severely restricted in maintaining employment due to a lack of proper transportation or not having an accessible work environment. The Social-environment domain lacks the depth necessary to measure the complex dynamic nature of the interaction of a person with disability and their environment. The US Institute of Medicine (IOM) recognizes the role of the environment as a mat or a cushion that can compensate for a person's disabilities [26]. The amount of support a person needs is a function of the level of disability and the strength/resilience of the physical and social environment. This domain does not fully address this relationship, potentially leading to misclassification of individuals and consequent misallocation of resources.

In summary, using the PAS-CS and disability grades as screening criteria for PAS is problematic. Since the PASCS greatly influences an individual's life by determining whether or not he/she is eligible for services, careful revision of evaluation items and content is required. 


\section{CONFLICT OF INTEREST}

No potential conflict of interest relevant to this article was reported.

\section{ACKNOWLEDGMENTS}

This research was supported in part by grant \# K12 HD055929 from the National Institutes of Health (NIH). Its contents are solely the responsibility of the authors and do not necessarily represent the official views of the NIH.

\section{REFERENCES}

1. Beatty PW, Richmond GW, Tepper S, DeJong G. Personal assistance for people with physical disabilities: consumer-direction and satisfaction with services. Arch Phys Med Rehabil 1998;79:674-7.

2. Misra S, Orslene LE, Walls RT. Personal Assistance Services (PAS) for workers with disabilities: Views and experiences of rehabilitation service providers. Disabil Stud Q 2007;27:31.

3. DeJong G, Wenker T. Attendant care as a prototype independent living service. Arch Phys Med Rehabil 1979;60:477-82.

4. Byun KH, Kim DK, Lee MJ, Won JP, Park HG, Lee W, et al. Modification of personal assistance service classification system. Osan: Hanshin University Industry Academic Cooperation Foundation; 2012.

5. LaPlante MP, Kaye HS, Kang T, Harrington C. Unmet need for personal assistance services: estimating the shortfall in hours of help and adverse consequences. J Gerontol B Psychol Sci Soc Sci 2004;59:S98-S108.

6. Allen SM, Mor V. The prevalence and consequences of unmet need: contrasts between older and younger adults with disability. Med Care 1997;35:1132-48.

7. Desai MM, Lentzner HR, Weeks JD. Unmet need for personal assistance with activities of daily living among older adults. Gerontologist 2001;41:82-8.

8. Lima JC, Allen SM. Targeting risk for unmet need: not enough help versus no help at all. J Gerontol B Psychol Sci Soc Sci 2001;56:S302-10.

9. Korean Ministry of Health and Welfare. 2017 Personal Assistance Services (Volume V). Sejong: Korean Ministry of Health and Welfare; 2017.
10. Kim SH, Byun YC, Lee SH, Cho HS, Kim CW, Lee SK, et al. Political research for implementing of personal assistance services. Sejong: Korea Institute for Health and Affairs; 2011.

11. Korea Employment Agency for the Disabled. 2016 Statistics for people with disabilities. Seongnam: Korea Employment Agency for the Disabled; 2016.

12. Kim SH, Lee YH, Hwang JH, Oh MA, Lee MK, Lee NH, et al. 2014 National Survey on People with Disabilities. Korean Institute for Health and Social Affairs; 2014.

13. The R Foundation. The R project for statistical computing (R 3.4.1) [Internet]. [place unknown]: The $\mathrm{R}$ Foundation; c2017 [cited 2018 Sep 15]. Available from: https://www.r-project.org/.

14. Rosseel Y. lavaan: an R package for structural equation modeling and more Version 0.4-9 (BETA) [Internet]. Ghent: Ghent University; 2011 [cited 2018 Sep 15]. Available from: http://byrneslab.net/classes/lavaan_ materials/lavaanIntroduction4-9.pdf.

15. Barton K. MuMIn: multi-model inference (Release 1.9.13) [Internet]. [place unknown]: The R Foundation; 2013 [cited 2018 Sep 15]. Available from: https:// cran.r-project.org/web/packages/MuMIn/index. html.

16. Hothorn T, Bretz F, Heiberger RM, Schuetzenmeister A, Scheibe S. multcomp: simultaneous inference in general parametric models (Release 1.4-2) [Internet]. [place unknown]: The R Foundation; 2016 [cited 2018 Sep 15]. Available from: https://cran.r-project.org/ web/packages/multcomp/index.html.

17. Komsta L, Novomestky F. moments: moments, cumulants, skewness, kurtosis and related tests (Release 0.14) [Internet]. [place unknown]: The R Foundation; 2015 [cited 2018 Sep 15]. Available from: https://cran. r-project.org/web/packages/moments/index.html.

18. Pena EA, Slate EH. gvlma: global validation of linear models assumptions (Release 0.0.0.2) [Internet]. [place unknown]: The R Foundation; 2014 [cited 2018 Sep 15]. Available from: https://cran.r-project.org/ web/packages/gvlma/index.html.

19. Ripley B, Venables B, Bates DM, Hornik K, Gebhardt A, Firth D. MASS: support functions and datasets for Venables and Ripley's MASS (Release 7.3.-48) [Internet]. [place unknown]: The R Foundation; 2017 [cited 2018 Sep 15]. Available from: https://cran.r-project. org/web/packages/MASS/index.html. 
20. Kleiber C, Zeileis A. AER: applied econometrics with $\mathrm{R}$ (Release 1.1.1) [Internet]. [place unknown]: The $\mathrm{R}$ Foundation; 2009 [cited 2018 Sep 15]. Available from: https://cran.r-project.org/web/packages/AER/index. html.

21. Wickham H, Francois R. dplyr: a grammar of data manipulation (Release 0.4.3) [Internet]. [place unknown]: The R Foundation; 2015 [cited 2018 Sep 15]. Available from: https://cran.r-project.org/web/packages/dplyr/index.html.

22. Seoul Information Communication Plaza. 2016 Personal assistance services [Internet]. Seoul: Seoul Metropolitan Government; 2016 [cited 2018 Sep 15]. Available from: http://opengov.seoul.go.kr/public/8151883.

23. Seoul Information Communication Plaza. Defini- tion and registration of the disabled [Internet]. Seoul: Seoul Metropolitan Government; 2016 [cited 2018 Sep 15]. Available from: http://disability.seoul.go.kr/registration/registration.jsp?Depth=1311.

24. van der Ploeg HP, van der Beek AJ, van der Woude LH, van Mechelen W. Physical activity for people with a disability: a conceptual model. Sports Med 2004;34:639-49.

25. Stancil MA. Physical activity and quality of life experienced by participants of a wheelchair basketball tournament [dissertation]. Gainesville: University of Florida; 2007.

26. Institute of Medicine. Enabling America: Assessing the role of rehabilitation science and engineering. Washington: National Academies Press; 1997. 